\title{
Perfil dos gerentes de Atenção Primária à Saúde de municípios de pequeno porte do norte do Paraná, Brasil
}

\author{
Profile of Primary Health Care managers \\ in small municipalities in the north of the state of Paraná, Brazil
}

Regina Hitomi Fukuda Ohira ${ }^{1}$

Luiz Cordoni Junior ${ }^{2}$

Elisabete de Fátima Polo de Almeida Nunes ${ }^{2}$

${ }^{1}$ Departamento Saúde e Educação, Universidade Estadual do Norte do Paraná. Campus Luiz Meneghel BR 369 Km 54, Vila Maria. 86.360-000 Bandeirantes PR Brasil. regina@uenp.edu.br

${ }^{2}$ Departamento de Saúde Coletiva, Centro de Ciências da Saúde, Universidade Estadual de Londrina.
Abstract The scope of this article was to establish the profile of Primary Health Care (PHC) managers in 49 small municipalities (up to 20,000 inhabitants) in the north of the state of Paraná. A semi-structured questionnaire was applied to 90 managers in a quantitative-based study. For statistical analysis the EpiInfo 3.5.1. program was used. The results of the manager profile showed that $91.1 \%$ were women, $83.3 \%$ nurses, $50 \%$ aged 21 to 30 years and $75.6 \%$ with specialization. Concerning the entry into employment, $70 \%$ were through public exam/selective test. Regarding professional experience, $40 \%$ had worked professionally for more than 5 years, $43.4 \%$ had been involved in PHC up to 5 years and $32.2 \%$ had been in the current primary health care unit for less than 1 year. Regarding specific training for performing a managerial role, $71.1 \%$ had not taken any courses; only $23.3 \%$ reported having taken a management course, though $37.7 \%$ had already acted as managers. The conclusion reached is that there is a lack of professionalism, training and institutionalization of the position for the performance of this function.

Key words Public health, Health service administration, Primary Health Care, Management, Health management
Resumo O presente artigo teve por objetivo caracterizar o perfil dos gerentes da atenção primária à saúde (APS) em 49 municípios de pequeno porte (até 20.000 habitantes) do norte do Paraná. Trata-se de um estudo de abordagem quantitativa no qual foi aplicado questionário semiestruturado para 90 gerentes. Para análise estatística, utilizouse o programa EpiInfo 3.5.1. O resultado do perfil dos gerentes apontou para $91,1 \%$ do sexo feminino, $83,3 \%$ enfermeiros, 50,0\% com idade predominante entre 21 e 30 anos e 75,6\% com especialização. Quanto ao ingresso no emprego, em 70,0\% foi por meio de concurso público/teste seletivo. Em relação à atuação profissional, 40,0\% atuavam há mais de 5 anos, 43,4\% estavam inseridos na APS há mais de 5 anos e 32,2\% na atual unidade básica de saúde há menos de 1 ano. Sobre treinamento específico para desempenho da função gerencial, $71,1 \%$ não fizeram nenhum curso, somente $23,3 \%$ relataram ter feito curso de gestão, mas 37,7\% já atuaram como gerentes. Conclui-se que falta profissionalização, capacitação e institucionalização do cargo para o desempenho desta função.

Palavras chave Saúde pública, Administração de serviços de saúde, Atenção Primária à Saúde, Gerência, Gestão em saúde 


\section{Introdução}

A partir da década de 1990, a área da saúde tem sido palco de reformas institucionais para implementar os princípios e as diretrizes do Sistema Único de Saúde (SUS). Destaca-se a Política Nacional de Atenção Primária à Saúde (PNAPS), com vistas a ampliar o acesso e desencadear a descentralização da política de saúde ${ }^{1}$. A descentralização, segundo Castro e Machado ${ }^{2}$, transferiu maior responsabilidade para a execução direta de ações e serviços de saúde principalmente aos municípios que estruturaram uma ampla rede de serviços de saúde no nível da atenção primária à saúde (APS), tornando-se os principais gestores da força de trabalho ${ }^{3}$.

Porém, os municípios que investiram em reformas na estrutura dos sistemas locais de saúde, mas que não enfrentaram o desafio de mudar o processo de trabalho e o modo hegemônico de se fazer saúde - caracterizados pela produção de procedimentos centrados no médico, medicalização e forma centralizada e burocrática de tomada de decisão - não conseguiram alterar de maneira substantiva a produção de saúde $e^{4}$.

Machado et $\mathrm{al}^{5}$ asseveram que o gestor é muito mais que um administrador. É autoridade sanitária em cada esfera de governo com duas dimensões de atuação: política, pois é designada pelo chefe do Executivo com responsabilidade de desenvolver o projeto de governo e técnica, desempenhada com conhecimento, habilidades e experiências da gestão pública em saúde para conduzir a política pautada pela legalidade, princípios do SUS e da reforma sanitária brasileira.

Os municípios de pequeno porte (MPP), definidos como aqueles com menos de 20 mil habitantes, representam $70,19 \%$ da totalidade dos municípios brasileiros e no Paraná $77,94 \%{ }^{6}$. A problemática apontada pelo Ministério das $\mathrm{Ci}$ dades para os MPP é a falta de estrutura para o planejamento e a baixa capacidade de gestão urbana para exercer a competência municipal constitucional ${ }^{7}$.

A gestão da saúde em MPP e seus agentes é complexa e pouco pesquisada empiricamente. Neste sentido, é importante realizar estudos para descrever quem são estes agentes, principalmente os que assumiram a gerência da APS. Para responder como os MPP estão se organizando nas unidades básicas de saúde, este estudo teve o objetivo de analisar o perfil dos gerentes que atuam na APS.

\section{Metodologia}

Trata-se de um estudo de abordagem quantitativa, realizado com 90 gerentes de unidades de atenção primária à saúde em 49 MPP do norte do Paraná. Foram investigados os municípios da $16^{\mathrm{a}}$, $17^{\mathrm{a}}$ e 18 ${ }^{\mathrm{a}}$ Regionais de Saúde (RS) da Secretaria de Estado da Saúde. Das 82 unidades de atenção primária existentes na área pesquisada, 77 foram contempladas. Portanto houve perda de 4 MPP com 5 unidades. Foram considerados perdas os gerentes que não devolveram os instrumentos até 10 dias após a visita no município para coleta de dados, e após 3 contatos telefônicos posteriores feitos pelos técnicos das respectivas RS. O critério de inclusão foi a indicação dos gerentes feita pelo secretário municipal de saúde de pelo menos 1 trabalhador de cada unidade de saúde da atenção primária, lembrando que em uma unidade pode haver até 2 coordenadores da estratégia saúde da família, indicados gerentes da APS.

Para a coleta de dados foi utilizado questionário semiestruturado aplicado in loco. Participaram da coleta técnicos das RS, graduandos de Enfermagem e Medicina, pós-graduandos dos programas de Mestrado e Doutorado em Saúde Coletiva da Universidade Estadual de Londrina, que participaram de um treinamento para homogeneização do entendimento das questões do instrumento de pesquisa.

As variáveis em estudo foram caracterização do perfil dos gerentes quanto a sexo, faixa etária, escolaridade, formação profissional, forma de ingresso no serviço, vínculo empregatício, jornada de trabalho, tempo de atuação no serviço e remuneração. $\mathrm{Na}$ análise estatística utilizou-se o programa EpiInfo 3.5.1 apresentando a frequência das variáveis comparada entre as três regionais em estudo.

A pesquisa foi norteada pelos princípios éticos contemplados na resolução do Conselho Nacional de Saúde no $196 / 96^{8}$, e o projeto foi submetido ao Comitê de Ética em Pesquisa (CEP) da UEL em outubro de 2009, com parecer favorável.

\section{Resultados}

\section{Características Sócio Demográficas dos Gerentes}

Observou-se a predominância do sexo feminino $(91,1 \%)$, com a faixa etária variando entre 21 e 62 anos, com mediana de 30 anos, prepon- 
derando entre 21 a 30 anos (50,0\%), conforme a Tabela 1.

Quanto à escolaridade, o menor grau dos participantes foi de 3 gerentes com ensino médio completo, 87 gerentes tinham ensino universitário. A categoria profissional predominante foi de 75 enfermeiros $(83,3 \%)$. Houve predomínio de pós-graduação representado por 68 participantes $(75,6 \%)$, sendo o grau máximo de formação no nível de especialização, não havendo doutor ou mestre entre os pesquisados.

\section{Formação dos Gerentes}

Na Tabela 2 estão elencados os cursos de especialização citados pelos gerentes. $\mathrm{O}$ curso de especialização em Saúde da Família foi o mais referido, por 19 gerentes $(21,1 \%)$, seguido de Saúde Coletiva, com 18 participantes (20,0\%). Não informaram o curso de pós-graduação realizado 28 participantes, representando $31,2 \%$.

Relacionado à formação para atuarem na função de gerentes da APS, 64 (71,1\%) dos participantes relataram não ter feito curso específico de gerência, e somente 21 (23,3\%) relataram ter feito curso nesta área.

\section{Contratação e Vínculo Empregatício}

Como demonstra a Tabela 3, quanto ao ingresso no emprego, $63(70,0 \%)$ relataram que o processo ocorreu por meio de concurso público/ teste seletivo, $25(27,8 \%)$ de outras formas e 2

Tabela 1. Idade, escolaridade, categorias profissionais e sexo dos gerentes da APS nos MPP da 16 ${ }^{\mathrm{a}}, 17^{\mathrm{a}}$ e $18^{\mathrm{a}}$ regionais de saúde do norte do Paraná, 2011.

\begin{tabular}{lrrrrr}
\hline \multicolumn{1}{c}{ Características } & $\mathbf{1 6}^{\mathbf{a}}$ & $\mathbf{1 7}^{\mathbf{a}}$ & $\mathbf{1 8}^{\mathbf{a}}$ & Total & $\mathbf{\%}$ \\
\hline Idade (em anos) & & & & & \\
$\quad 21$ a 30 & 9 & 18 & 18 & 45 & 50,0 \\
31 a 40 & 9 & 10 & 5 & 24 & 26,7 \\
$\quad 41$ e mais & 5 & 8 & 5 & 18 & 20,0 \\
$\quad$ Não informado & 2 & - & 1 & 3 & 3,3 \\
Escolaridade & & & & & \\
$\quad$ Ensino Médio & 1 & - & 2 & 3 & 3,3 \\
$\quad$ Ensino Superior & 6 & 6 & 7 & 19 & 21,1 \\
$\quad$ Pós-graduação & 18 & 30 & 20 & 68 & 75,6 \\
Categoria Profissional & & & & & \\
$\quad$ Enfermeiros & 21 & 36 & 18 & 75 & 83,3 \\
$\quad$ Outros & 4 & - & 11 & 15 & 16,7 \\
Sexo & & & & & \\
Feminino & 21 & 34 & 27 & 82 & 91,1 \\
Masculino & 4 & 2 & 2 & 8 & 8,9 \\
\hline
\end{tabular}

Tabela 2. Pós-graduação dos gerentes da 16a $17^{\mathrm{a}}$ e $18^{\mathrm{a}}$ Regional de Saúde nos MPP na região norte do PR, 2011.

\begin{tabular}{|c|c|c|c|c|c|}
\hline Características & $16^{\mathrm{a}}$ & $17^{a}$ & $18^{\mathrm{a}}$ & $\mathbf{N}$ & $\%$ \\
\hline \multicolumn{6}{|l|}{ Especialização } \\
\hline Saúde da Família & 5 & 8 & 6 & 19 & 21,1 \\
\hline Saúde Coletiva & 1 & 11 & 6 & 18 & 20,0 \\
\hline Enfermagem do Trabalho & 4 & 5 & 2 & 11 & 12,3 \\
\hline Enfermagem Obstétrica & 2 & 3 & 1 & 6 & 6,7 \\
\hline Urgência e Emergência & 1 & 1 & 2 & 4 & 4,4 \\
\hline Saúde do Trabalhador & 1 & - & 1 & 2 & 2,2 \\
\hline Vigilância em Saúde & 2 & - & - & 2 & 2,2 \\
\hline Outras & 3 & 2 & 1 & 6 & 6,7 \\
\hline Não informado & 6 & 6 & 10 & 22 & 24,4 \\
\hline \multicolumn{6}{|c|}{ Conclusão da especialização (em anos) } \\
\hline Menos de 1 & 2 & 3 & 1 & 6 & 6,7 \\
\hline$>1$ a 3 & 7 & 7 & 6 & 20 & 22,2 \\
\hline$>3$ a 5 & 3 & 11 & 6 & 20 & 22,2 \\
\hline$>5$ & 4 & 5 & 4 & 13 & 14,4 \\
\hline Sem especialização & 1 & - & 2 & 03 & 3,3 \\
\hline Não informado & 8 & 10 & 10 & 28 & 31,2 \\
\hline \multicolumn{6}{|l|}{ Curso de gerência } \\
\hline $\operatorname{Sim}$ & 7 & 12 & 2 & 21 & 23,3 \\
\hline Não & 17 & 24 & 23 & 64 & 71,1 \\
\hline Não informado & 1 & 1 & 3 & 5 & 5,6 \\
\hline
\end{tabular}


Tabela 3. Modalidade de seleção, de contratação, remuneração, carga horária semanal e vínculo dos gerentes da atenção primária à saúde dos MPP da 16ª 17a e 18 $\mathrm{RS}$ do norte do Paraná, 2011.

\begin{tabular}{|c|c|c|c|c|c|}
\hline & $16^{\mathrm{a}}(\mathrm{n}=25)$ & $17^{a}(n=36)$ & $18^{\mathrm{a}}(\mathrm{n}=29)$ & n (90) & $\%(100)$ \\
\hline \multicolumn{6}{|l|}{ Ingresso no Emprego } \\
\hline Concurso Público/Teste Seletivo & 13 & 28 & 22 & 63 & 70,0 \\
\hline Outras formas & 12 & 8 & 5 & 25 & 27,8 \\
\hline Não informado & - & - & 2 & 2 & 2,2 \\
\hline \multicolumn{6}{|l|}{ Modalidade de Contratação } \\
\hline Consolidação das Leis Trabalhista (CLT) & 11 & 17 & 12 & 40 & 44,4 \\
\hline Estatuto do Servidor Público & 8 & 12 & 12 & 32 & 35,5 \\
\hline Convênio/Terceirizado & 3 & 5 & 3 & 11 & 12,3 \\
\hline Outro & 3 & 1 & 2 & 6 & 6,7 \\
\hline Não informado & - & 1 & - & 1 & 1,1 \\
\hline \multicolumn{6}{|l|}{ Remuneração Mensal ( $\left.{ }^{*} \mathrm{SM}\right)$} \\
\hline 1 a $3 \mathrm{SM}$ & 14 & 13 & 9 & 36 & 40,0 \\
\hline$>3$ a $5 \mathrm{SM}$ & 10 & 15 & 11 & 36 & 40,0 \\
\hline$>5 \mathrm{SM}$ & - & 2 & 2 & 04 & 4,4 \\
\hline Não informado & 1 & 6 & 7 & 14 & 15,6 \\
\hline \multicolumn{6}{|l|}{ Carga Horária Contratada Semanal } \\
\hline 40hs & 23 & 30 & 25 & 78 & 86,7 \\
\hline 20 a $38 \mathrm{hs}$ & 2 & 5 & 1 & 8 & 8,9 \\
\hline Não informado & - & 1 & 3 & 4 & 4,4 \\
\hline \multicolumn{6}{|l|}{ Outro Vínculo de Trabalho } \\
\hline $\operatorname{Sim}$ & 8 & 1 & 1 & 10 & 11,1 \\
\hline Não & 17 & 35 & 28 & 80 & 88,9 \\
\hline
\end{tabular}

*1 Salário Mínimo $=\mathrm{R} \$ 510,00$.

não informaram (2,2\%). Dos 25 gerentes que responderam por outras formas de contratação, 19 $(21,1 \%)$ relataram por indicação, $3(3,3 \%)$ por análise de curriculum vitae, 2 (2,2\%) por entrevista e $1(1,1 \%)$ não especificou a forma de ingresso. Quanto à modalidade de contratação, 40 $(44,4 \%)$ foram pela Consolidação das Leis do Trabalho (CLT) com vínculo municipal, 32 (35,5\%) pelo estatuto do servidor público, 11 $(12,3 \%)$ por convênio/terceirização, $6(6,7 \%)$ citaram outras formas de contratação e 1 participante $(1,1 \%)$ não respondeu (Tabela 3). Quando questionados sobre o recebimento de algum incentivo além do salário, 36 participantes (40\%) responderam que não, 49 responderam $(54,4 \%)$ que sim, porém não é específico para a função gerencial.

Questionados ainda sobre a indicação para o cargo de gerente, 85 participantes $(94,4 \%)$ responderam, sendo que destes, 61 foram indicados pelo secretário municipal de saúde representando $67,7 \%, 24$ participantes $(26,7 \%)$ indicados por outras formas não especificadas e $5(5,6 \%)$ não responderam.
Em relação à existência de Plano de Cargos, Carreiras e Salários (PCCS) no município, dos 83 respondentes, 77 gerentes $(74,4 \%)$ relataram que não há PCCS instituído no município, 13 $(14,4 \%)$ responderam que já está instituído e 3 (3,3\%) não souberam informar.

Quanto às faixas de remuneração mensal, foi utilizado como base o valor bruto do salário mínimo nacional (SM) de R\$510,00. Não responderam 14 participantes $(15,6 \%)$ e entre os que relataram, a remuneração variou entre $\mathrm{R} \$$ 670,00 a $\mathrm{R} \$ 2.880,00$ com a mediana de $\mathrm{R} \$$ $1.600,00$, sendo na faixa de 1 a 3 SM (de R $\$ 670,00$ a R $\$ 1.530,00)$ com 36 dos participantes $(40,0 \%)$, entre 3 a 5 SM também com mesmo número e percentual e $4(4,4 \%)$ recebiam acima de $5 \mathrm{SM}$ ( R\$2.550,00 a R\$2.880,00).

Relativamente à jornada de trabalho, predominou a contratação da carga horária de 40 horas semanais com 78 participantes $(86,7 \%)$, seguida de 8 participantes $(8,9 \%)$ com contratos de 20 a 38 horas e $4(4,4 \%)$ não responderam. Relacionado ao número de vínculos de trabalho, 80 participantes $(88,9 \%)$ tinham somente 1 vínculo, $10(11,1 \%)$ possuíam outro vínculo. 
anos de atuação seguido de 19 (21,1\%) entre 3 a 5 anos com predominância na $18^{\mathrm{a}}$ Regional de Saúde, 9 participantes $(10,0 \%)$ com até 1 ano de atuação e 6 participantes $(6,7 \%)$ não informaram (Tabela 4).

Quanto ao tempo de atuação na APS, 39 participantes $(43,4 \%)$ responderam atuar em período superior a 5 anos, $23(25,5 \%)$ num período entre 1 a 3 anos seguido de $17(18,9 \%)$ entre 3 a 5 anos e $9(10,0 \%)$ participantes com até 1 ano de atuação. Deixaram de informar 2 participantes $(2,2 \%)$. Relacionado ao tempo de atuação enquanto gerente na atual UBS, predominou o período menor que 1 ano, com 29 participantes (32,2\%) seguido de 24 gerentes $(26,7 \%)$ no período entre 1 a 3 anos, 17 participantes (18,9\%)

num período acima de 5 anos, 13 participantes $(14,4 \%)$ entre 3 a 5 anos e 7 participantes $(7,8 \%)$ não informaram. Dos participantes da pesquisa, $53(59 \%)$ não atuaram como gerentes em outra UBS e $34(37,7 \%)$ já atuaram.

Dentre as outras funções exercidas concomitantemente pelos gerentes dos MPP, 51 (56,7\%) responderam que sim. Citaram: eram responsáveis pela vigilância epidemiológica, realizavam serviços assistenciais (enfermeiros do PSF, fisioterapeuta, enfermeira obstétrica), assinavam como responsáveis técnicos da unidade e atuavam como enfermeiras da UBS. Também exerciam a coordenação dos programas desenvolvidos na UBS, tinham a responsabilidade pela alimentação do sistema de informação, responsabilidade pela ouvidoria da saúde e respondiam pela vigilância sanitária. Ainda foram citados: presidente do conselho municipal de saúde, responsável pela sala de vacina, coordenador da dengue, agricultor, secretaria do CMS e presidência da comissão de licitação.

Tabela 4. Experiência profissional, na atenção primária e gerência dos gerentes da $16^{\mathrm{a}}, 17^{\mathrm{a}}$ e $18^{\mathrm{a}}$ Regional de Saúde nos MPP na região norte do PR, 2011.

\begin{tabular}{|c|c|c|c|c|c|}
\hline Características & $16^{\mathrm{a}}$ & $17^{a}$ & $18^{\mathrm{a}}$ & $\mathbf{N}$ & $\%$ \\
\hline \multicolumn{6}{|l|}{ Tempo de atuação profissional (anos) } \\
\hline Até $1>$ & 4 & 3 & 2 & 9 & 10,0 \\
\hline 1 a 3 & 6 & 8 & 6 & 20 & 22,2 \\
\hline$>3$ a 5 & 3 & 7 & 9 & 19 & 21,1 \\
\hline$>5$ & 11 & 15 & 10 & 36 & 40,0 \\
\hline Não informado & 1 & 3 & 2 & 6 & 6,7 \\
\hline \multicolumn{6}{|l|}{ Tempo de atuação na APS (anos) } \\
\hline Até 1 & 3 & 3 & 3 & 9 & 10,0 \\
\hline$>1$ a 3 & 6 & 10 & 7 & 23 & 25,5 \\
\hline$>3$ a 5 & 3 & 8 & 6 & 17 & 18,9 \\
\hline$>5$ & 12 & 15 & 12 & 39 & 43,4 \\
\hline Não informado & 1 & - & 1 & 2 & 2,2 \\
\hline \multicolumn{6}{|c|}{ Tempo de atuação na gerência nesta unidade (anos) } \\
\hline Até 1 & 9 & 12 & 8 & 29 & 32,2 \\
\hline$>1$ a 3 & 7 & 9 & 8 & 24 & 26,7 \\
\hline$>3$ a 5 & 5 & 7 & 1 & 13 & 14,4 \\
\hline$>5$ & 2 & 8 & 7 & 17 & 18,9 \\
\hline Não informado & 2 & - & 5 & 7 & 7,8 \\
\hline \multicolumn{6}{|l|}{ Gerência em outra unidade } \\
\hline Sim & 11 & 11 & 12 & 34 & 37,7 \\
\hline Não & 13 & 25 & 15 & 53 & 59 \\
\hline Não informado & 1 & - & 2 & 3 & 3,3 \\
\hline \multicolumn{6}{|l|}{ Exerce outra função } \\
\hline Sim & 13 & 23 & 16 & 51 & 56,7 \\
\hline Não & 11 & 11 & 12 & 34 & 37,7 \\
\hline Não informado & 1 & 2 & 1 & 5 & 5,6 \\
\hline
\end{tabular}




\section{Discussão}

A categoria profissional predominante na função de gerentes da APS era a de enfermeiro. Fernandes et al. ${ }^{9}$ citam que esta categoria é uma das mais mobilizadas na saúde para o gerenciamento das UBS, utilizando a "descentralização administrativa, comunicação informal e criatividade" para exercê-lo. Dentre os profissionais que atuam na área da saúde, "os enfermeiros, em sua prática cotidiana demonstram maior interesse, vocação e preparo para assumir as funções de gestor" ${ }^{10}$. A característica no trabalho de enfermagem são bens produzidos e consumidos no ato da produção, exigindo a capacidade de desempenhar o papel de gerente nos serviços de saúde, dentro de uma perspectiva participativa, onde o objetivo é alcançado pelo esforço coletivo e não pela união de esforços individuais ${ }^{11}$.

O exercício profissional dessa prática gerencial é regulamentado pelo Conselho Federal de Enfermagem (COFEN) por meio da Resolução COFEN 194/1997'12 que oficializa a participação do enfermeiro na gerência geral, informando: "O enfermeiro pode ocupar em qualquer esfera, cargo de direção geral nas instituições de saúde, públicas e privadas, cabendo-lhe, ainda, privativamente, a direção dos serviços de enfermagem".

Também as Diretrizes Nacionais Curriculares (DNC) para a Graduação em Enfermagem ${ }^{13}$ incluem os conteúdos de administração/gestão entre aqueles considerados imprescindíveis ao desenvolvimento das habilidades e competências profissionais, sendo a única dentre as congêneres de graduação da área da saúde em que esses conteúdos estão presentes.

Relacionados com as características sociodemográficas e de formação, os resultados deste estudo têm semelhança com a pesquisa realizada por Gomes et al. ${ }^{14}$ no município de Cajuri (MG) que constatou média de idade de 35,2 anos, predominância do sexo feminino e predomínio de pós-graduados. As autoras observaram que $85,7 \%$ tinham pós-graduação, dos quais $42,8 \%$ haviam se aperfeiçoado ou ainda estavam realizando cursos na área da Saúde Pública.

Quanto ao ingresso no emprego, que neste estudo foi predominantemente por concurso público ou teste seletivo, os resultados foram melhores que os de Junqueira et al. ${ }^{15}$ no qual apenas 20\% ingressaram por meio de concurso público, predominando a "incorporação arcaica do empreguismo, clientelismo e patrimonialismo, como, por exemplo, a indicação política". Mendonça et al. ${ }^{16}$ constataram que especialmen- te no nível municipal os contratos precários estão sendo substituídos por quadros próprios por meio da realização de concurso público.

Segundo Junqueira et al. ${ }^{15}$ houve mudanças importantes na proporção de empregos públicos citando que "no ano de 1980, a esfera federal detinha mais de $42 \%$ do total das vagas de emprego, e a esfera municipal, apenas 16,2\%; em 2005, os empregos da esfera municipal passam a representar $68 \%$, e os federais, apenas 7,3\%", lembrando o número de MPP brasileiros como grandes empregadores desta mão de obra.

A modalidade de contratação pela Consolidação das Leis do Trabalho segue o recomendado pelo Artigo 37 da Constituição Federal do Brasil (1988). É importante esta reflexão, pois neste estudo $35,5 \%$ dos profissionais referiram contrato pelo Estatuto do Servidor Público que somado com a modalidade de CLT resulta em $79,9 \%$. Os gerentes contratados por outras formas têm vínculo precário e Victora et al. ${ }^{17}$ chamam atenção sobre os "contratos especiais" dos profissionais da estratégia saúde da família, permitindo a contratação e a demissão com mais facilidade do que os concursados, pagamento de salários superiores, porém com ausência de estabilidade e benefícios resultando em insatisfação e rotatividade de pessoal, o que leva a uma descontinuidade do cuidado.

A não existência de PCCS, associada aos fatos de a indicação para a gerência ser feita predominantemente pelo secretário de saúde e ausência de incentivo salarial para a função permite inferir que o cargo de gerente não é formalizado.

Pelo pequeno percentual de gerentes dos MPP pesquisados terem feito cursos de gerência, é necessário o incentivo para realizá-los, pois a qualificação/capacitação do profissional de saúde é um dos caminhos para a profissionalização da gestão. Sinalizado neste estudo que é necessário pensar na instituição do contrato formal para os gerentes dos MPP, pois alguns foram indicados pelo gestor municipal durante a pesquisa in loco pela necessidade de conduzir o trabalho dos pesquisadores na aplicação do instrumento.

Neste sentido, os municípios deverão se estruturar e organizar técnica e administrativamente porque é a esfera de governo mais próxima da população. Para firmar a perplexidade dos pesquisadores, segue uma frase da entrevista de Campos realizada por De Lavor ${ }^{18}$ que diz que "no SUS, até hoje, diretores de unidades básicas e de hospitais são cargos de confiança de secretários. Isso é um absurdo. No mundo inteiro não é mais assim". 
Ressalte-se que o gestor necessita aliar conhecimentos, habilidades e experiências na gestão pública para conduzir a política de saúde pautada nos princípios e na legalidade do SUS 5 . Tais qualidades requerem uma imersão formal nas funções que desenvolvem.

Quanto à remuneração mensal, neste estudo a mediana foi de $\mathrm{R} \$ 1.600,00$, abaixo do estudo de Gomes et al. ${ }^{14}$ que obteve R\$2.670,00. Estes autores relatam que baixos salários são entraves para o desenvolvimento das características da APS. Estes salários relativamente baixos talvez se devam, em parte ao fato de a maioria serem mulheres, que costumam ter salários inferiores aos dos homens como assinala Barata ${ }^{19}$.

$\mathrm{Na}$ atual pesquisa, a carga horária de trabalho contratada dos gerentes pode ser justificada provavelmente porque há vários enfermeiros que são da equipe de saúde da família e foram contratados segundo as diretrizes da estratégia.

Para a profissionalização da gestão, o que chama atenção é o baixo percentual dos gerentes que fizeram curso para exercerem a função e cinco gerentes consideraram a graduação em enfermagem como curso preparatório. Mendonça et al. ${ }^{16}$ citam dois programas que estão contribuindo diretamente para que a gestão do trabalho no SUS se qualifique: o Programa de Capacitação Gerencial para o Sistema Único de Saúde (PROGESUS), voltado para a qualificação de gestores, com vistas a enfatizar o importante papel dos gerentes de articulador de ações que resultem na melhoria da qualidade de atenção, a partir da estruturação/qualificação dos serviços de saúde e o programa da Universidade Aberta do Sistema Único de Saúde (UNASUS), criado em junho de 2008, que facilita o funcionamento de uma rede colaborativa de instituições acadêmicas, serviços de saúde e gestão do SUS, destinada a atender necessidades de formação e educação permanente em saúde em áreas estratégicas.

O objetivo maior de se fazer gestão é a busca de um mundo diferente. Na saúde menos doença, menos mortes, mais qualidade de vida e isto é tarefa dos gerentes de saúde propiciando e facilitando a organização dos serviços e da sociedade. É necessário "uma caixa de ferramenta, entendida como o conjunto de saberes, para conformação do cuidado, para compreender os problemas de saúde e enfrentá-lo adequadamente". Portanto, enquanto gerentes na atenção primária, este espaço se abre e com as práticas gerenciais possibilita combinar a produção de atos cuidadores de maneira eficaz com conquistas de resultados ${ }^{20}$.

\section{Considerações finais}

O estudo realizou uma descrição do perfil dos gerentes nos MPP de três regionais de saúde no norte do Paraná. Vários estudos descrevem estas questões nos municípios médios e grandes, onde a organização do setor da saúde é diferenciada pelo contingente de profissionais que atuam especificamente nesta função, o que não acontece nos MPP onde a escassez de profissionais é visível, sendo que em muitos, quem atua como gerente é o único que conduz todo serviço e as ações de saúde.

Nos resultados quanto ao perfil dos sujeitos do estudo, não se pode negar os avanços no sistema de saúde constatados na pesquisa, principalmente relacionados ao vínculo de contratação que possibilita a fixação dos profissionais de saúde para compor o quadro próprio da saúde. Quanto à qualificação, o trabalho indicou que os gerentes da APS nos MPP pesquisados não a possuem. Também têm pouca experiência no cargo que ocupam e atuam. Devido à importância da função gerencial, as grandes empresas privadas preparam e formam, durante anos, o profissional para exercer esta função, o que não acontece no setor público. Este trabalho evidencia a necessidade de qualificação do gerente como um desafio a ser enfrentado.

Para as secretarias municipais de saúde dos MPP pesquisados é urgente formalizar o cargo de gerentes nas UBS, estabelecer suas competências e promover discussão sobre o perfil gerencial e seus reflexos no processo de reorientação do modelo de organização e atenção à saúde. É necessário também a valorização dos serviços públicos, salários mais justos e condizentes com as responsabilidades exigidas.

Para que o SUS seja adequadamente implementado, a gestão deve ser um tema sempre presente nas discussões de todas as esferas de governo, principalmente no nível local porque depende destas instâncias para a mudança do panorama nacional. 


\section{Colaboradores}

RHF Ohira, L Cordoni Junior e EFPA Nunes participaram de todas as etapas da pesquisa.

\section{Agradecimentos}

À Fundação Araucária de Apoio ao Desenvolvimento Científico e Tecnológico do Estado do Paraná pelo financiamento da pesquisa.

\section{Referências}

1. Lotufo M, Miranda AS de. Sistemas de direção e práticas de gestão governamental em secretarias estaduais de saúde. Rev adm publica. 2007; 41(6):11431163.

2. Castro ALB de, Machado CV. A política de atenção primária à saúde no Brasil: notas sobre a regulação e o financiamento federal. Cad Saude Publica 2010; 26(4):693-705.

3. Levcovitz E, Lima LD de, Machado CV. Política de saúde nos anos 90: relações intergovernamentais e o papel das normas operacionais básicas. Cien Saude Colet 2001; 6(2):269-291.

4. Pimenta AL. Saúde de Amparo: a construção de espaços coletivos de gestão 2002-2005 [tese]. Campinas (SP): Faculdade de Ciências Médicas; 2006.

5. Machado CV, Lima LD, Baptista TW de F. Princípios organizativos e instâncias de gestão do SUS. In: Gondim R, Gabrois V, Mendes W, organizadores. Qualificação de gestores no SUS. Rio de Janeiro: EAD, Ensp; 2011. p. 47-72.

6. Instituto Brasileiro de Geografia e Estatística (IBGE). Cidades: estimativa de população. [página na Internet] 2010. [acessado 2013 nov 12]. Disponível em: http://www.ibge.gov.br/cidadesat/topwindow.htm?1

7. Brasil. Ministério das Cidades. Plano diretor em municípios de pequeno porte. [página na Internet]. [acessado 2013 nov 12]. Disponível em: http://portal.cnm.org.br/ sites/8800/8875/download/2_dia/PlanoDiretorem Municipiosdepequenoporte_Modificada.pdf

8. Brasil. Ministério da Saúde (MS). Conselho Nacional de Saúde. Resolução no ${ }^{\circ} 196$ de 10 de outubro de 1996. Diretrizes e Normas Regulamentadoras de Pesquisas Envolvendo Seres Humanos. Diário Oficial da União 1996; 16 out.

9. Fernandes MC, Barros AS, Silva LMA, Nóbrega MFB, Silva MRF, Torres RAM. Análise da atuação do enfermeiro na gerência de unidades básicas de saúde. Rev bras enferm. 2010; 63(1):11-15.

10. Coatto A, Traldi MC. Competências gerenciais do enfermeiro na atenção primária. Cad saúde colet. 2011; 52(8):180-186.

11. Weirich CF, Munari DB, Mishima SM, Bezzerra ALQ. O trabalho gerencial do enfermeiro na rede básica de saúde. Texto \& contexto enferm. 2009; 18(2):249-257.

12. Conselho Federal de Enfermagem. Resolução COFEN-194, de 18 de fevereiro de 1997. [página na Internet]. [acessado 2013 nov 12]. Disponível em: http://novo.portalcofen.gov.br/resoluo-cofen1941997_4251.html
13. Brasil. Conselho Nacional de Educação. Câmara de Educação Superior. Resolução CNE/CES no 3, de 7 de novembro de 2001. Institui Diretrizes Curriculares Nacionais do Curso de Graduação em Enfermagem. Diário Oficial da União 2001; 9 nov.

14. Gomes KO, Cotta RMM, Araújo RMA, Cherchiglia ML, Martins TCP. Atenção primária à saúde: a "menina dos olhos" do SUS: sobre as representações sociais dos protagonistas do Sistema Único de Saúde. Cien Saude Colet 2011; 16(Supl. 1):881-892.

15. Junqueira TS, Cotta RMM, Gomes RC, Silveira SFR, Siqueira-Batista R, Pinheiro TMM, Sampaio RF. As relações laborais no âmbito da municipalização da gestão em saúde e os dilemas da relação expansão/ precarização do trabalho no contexto do SUS. Cad Saude Publica 2010; 26(5):918-928.

16. Mendonça MHM, Martins MIC,Giovanella L, Escorel S. Desafios para gestão do trabalho a partir de experiências exitosas de expansão da estratégia de saúde da família. Cien Saude Colet 2010; 15(5):23552365.

17. Victora CG, Barreto ML, Leal MC, Monteiro CA, Schmidt MI, Paim J, Bastos FI, Almeida C, Bahia L, Travassos C, Reichenheim M, Barros FC, the Lancet Brazil Series Working Group. Condições de saúde e inovações nas políticas de saúde no Brasil: o caminho a percorrer. The Lancet 2011; 377(9782): 2042-2053.

18. De Lavor A. Entrevista: Gastão Wagner de Sousa Campos: é preciso um novo pacto da sociedade pelo SUS. Radis dados. 2011; 108:18-21.

19. Barata RB. Relações de gênero e saúde: desigualdade ou discriminação? In: Barata RB. Como e por que as desigualdades sociais fazem mal à saúde. Rio de Janeiro: Fiocruz; 2009.

20. Merhy EE. Um dos grandes desafios para os gestores do SUS: apostar em novos modos de fabricar os modelos de atenção. In: Merhy EE, Magalhães Junior HM, Rimoli J, Franco TB, Bueno WS. O trabatho em saúde: olhando e experienciando o SUS no cotidiano. São Paulo: Hucitec; 2007. p. 15-35.

Artigo apresentado em 25/11/2012

Aprovado em 30/04/2013

Versão final apresentada em 17/05/2013 